\title{
Fractional integral associated to Schrödinger operator on the Heisenberg groups in central generalized Morrey spaces
}

\author{
Ahmet Eroglua,*, Tahir Gadjiev ${ }^{\mathrm{b}}$, Faig Namazov ${ }^{\mathrm{c}}$ \\ ${ }^{a}$ Nigde Omer Halisdemir University, Department of Mathematics, Nigde, Turkey. \\ ${ }^{b}$ Institute of Mathematics and Mechanics of NAS of Azerbaijan, AZ1141 Baku, Azerbaijan. \\ ${ }^{c}$ Baku State University, AZ1141 Baku, Azerbaijan.
}

Communicated by M. Alessandra Ragusa

\begin{abstract}
Let $L=-\Delta_{\mathbb{H}_{n}}+V$ be a Schrödinger operator on the Heisenberg groups $\mathbb{H}_{n}$, where the non-negative potential $V$ belongs to the reverse Hölder class $\mathrm{RH}_{\mathrm{Q} / 2}$ and $\mathrm{Q}$ is the homogeneous dimension of $\mathbb{H}_{n}$. Let $\mathrm{b}$ belong to a new $B M O_{\theta}\left(\mathrm{H}_{n}, \rho\right)$ space, and let $\mathcal{J}_{\beta}^{\mathrm{L}}$ be the fractional integral operator associated with $\mathrm{L}$. In this paper, we study the boundedness of the operator $\mathcal{J}_{\beta}^{\mathrm{L}}$ and its commutators $\left[b, \mathcal{J}_{\beta}^{\mathrm{L}}\right]$ with $\mathrm{b} \in \mathrm{BMO}_{\theta}\left(\mathbb{H}_{n}, \rho\right)$ on central generalized Morrey spaces $\operatorname{LM}_{p, \varphi}^{\alpha, V}\left(\mathbb{H}_{n}\right)$ and generalized Morrey spaces $M_{p, \varphi}^{\alpha, V}\left(\mathbb{H}_{n}\right)$ associated with Schrödinger operator. We find the sufficient conditions on the pair $\left(\varphi_{1}, \varphi_{2}\right)$ which ensures the boundedness of the operator $\mathcal{J}_{\beta}^{L}$ from $\operatorname{LM}_{p^{\prime}, \varphi_{1}}^{\alpha, V}\left(\mathbb{H}_{n}\right)$ to $L_{M_{q}, \varphi_{2}}^{\alpha, V}\left(\mathbb{H}_{n}\right)$ and from $M_{p, \varphi_{1}}^{\alpha, V}\left(\mathbb{H}_{n}\right)$ to $M_{q, \varphi_{2}}^{\alpha, V}\left(\mathbb{H}_{n}\right), 1 / p-1 / q=\beta / Q$. When $b$ belongs to $\mathrm{BMO}_{\theta}\left(\mathbb{H}_{n}, \rho\right)$ and $\left(\varphi_{1}, \varphi_{2}\right)$ satisfies some conditions, we also show that the commutator operator $\left[\mathrm{b}, \mathrm{J}_{\beta}^{\mathrm{L}}\right]$ is bounded from $L M_{p, \varphi_{1}}^{\alpha, V}\left(\mathbb{H}_{n}\right)$ to $\operatorname{LM}_{q, \varphi_{2}}^{\alpha, V}\left(\mathbb{H}_{n}\right)$ and from $M_{p, \varphi_{1}}^{\alpha, V}$ to $M_{q, \varphi_{2}}^{\alpha, V}, 1 / p-1 / q=\beta / Q$.
\end{abstract}

Keywords: Schrödinger operator, Heisenberg group, central generalized Morrey space, fractional integral, commutator, BMO. 2010 MSC: 22E30, 35J10, 42B35, 47H50.

(C)2018 All rights reserved.

\section{Introduction}

Heisenberg groups, in discrete and continuous versions, appear in many parts of mathematics, including Fourier analysis, several complex variables, geometry, and topology. We state some basic results about Heisenberg group. More detailed information can be found in $[6,11,12]$ and the references therein.

Let us consider the Schrödinger operator on Heisenberg group $\mathbb{H}_{n}$

$$
L=-\Delta_{\mathbb{H}_{n}}+V \text { on } \mathbb{H}_{n}, n \geqslant 3,
$$

\footnotetext{
*Corresponding author

Email addresses: aeroglu@ohu.edu.tr (Ahmet Eroglu), tgadjiev@mail.az (Tahir Gadjiev), f-namazov@mail.ru (Faig Namazov)
}

doi: $10.22436 /$ jnsa.011.08.05

Received: 2018-04-05 Revised: 2018-04-22 Accepted: 2018-04-26 
where $V$ is a non-negative, $V \neq 0$, and belongs to the reverse Hölder class $R H_{q}$ for some $q \geqslant Q / 2$, i.e., there exists a constant $C>0$ such that the reverse Hölder inequality

$$
\left(\frac{1}{|B(g, r)|} \int_{B(g, r)} V^{q}(h) d h\right)^{1 / q} \leqslant \frac{C}{|B(g, r)|} \int_{B(g, r)} V(h) d h
$$

holds for every $g \in \mathbb{H}_{n}$ and $0<r<\infty$, where $B(g, r)$ denotes the ball centered at $g$ with radius $r$. In particular, if $V$ is a nonnegative polynomial, then $V \in R H_{\infty}$. Obviously, $R H_{q_{2}} \subset R H_{q_{1}}$, if $q_{2}>q_{1}$. The reverse Hölder class $\mathrm{RH}_{\mathrm{q}}$ have property, that is, if $\mathrm{V} \in \mathrm{RH}_{\mathrm{q}}$, then $\mathrm{V} \in \mathrm{RH}_{\mathrm{q}+\epsilon}$ for some $\epsilon>0$.

We define the auxiliary function $0<\rho(g)<\infty$ for a given potential $V \in \mathrm{RH}_{\mathrm{q}}$ with $\mathrm{q} \geqslant \mathrm{Q} / 2$,

$$
\rho(g):=\frac{1}{m_{V}(g)}=\sup _{r>0}\left\{r: \frac{1}{r^{n-2}} \int_{B(g, r)} V(h) d h \leqslant 1\right\}
$$

for any $\mathrm{g} \in \mathbb{H}_{\mathrm{n}}$ (for example, see [26]).

The BMO space $\mathrm{BMO}_{\theta}\left(\mathbb{H}_{n}, \rho\right)$ associated with Schrödinger operator with $\theta \geqslant 0$ is defined as a set of all locally integrable functions $b$ such that

$$
\frac{1}{|B(g, r)|} \int_{B(g, r)}\left|b(h)-b_{B}\right| d h \leqslant C\left(1+\frac{r}{\rho(g)}\right)^{\theta}
$$

for all $g \in \mathbb{H}_{n}$ and $r>0$, where $b_{B}=\frac{1}{|B|} \int_{B} b(h) d h$ (see [4]). A norm for $b \in B M O_{\theta}\left(\mathbb{H}_{n}, \rho\right)$, denoted by $[b]_{\theta}$, is given by the infimum of the constants in the inequalities above. Clearly, $\mathrm{BMO}\left(\mathbb{H}_{\mathrm{n}}\right) \subset$ $\mathrm{BMO}_{\theta}\left(\mathrm{H}_{n}, \rho\right)$. We give the definition of central (local) and global generalized Morrey spaces (including weak version) associated with Schrödinger operator, which is introduced by Guliyev in [14] on the Euclidean setting (see also [1, 2, 4, 20, 28]).

Definition 1.1. Let $\varphi(r)$ be a positive measurable function on $(0, \infty), 1 \leqslant p<\infty, \alpha \geqslant 0$, and $V \in \mathrm{RH}_{\mathrm{q}}$, $q \geqslant 1$. We denote by $M_{p, \varphi}^{\alpha, V}=M_{p, \varphi}^{\alpha, V}\left(\mathbb{H}_{n}\right), L_{p, \varphi}^{\alpha, V}=L_{p, \varphi}^{\alpha, V}\left(H_{n}\right)$ the generalized Morrey space, the central generalized Morrey space associated with Schrödinger operator, the spaces of all functions $f \in L_{\text {loc }}^{p}\left(\mathbb{H}_{n}\right)$ with finite quasinorms

$$
\begin{aligned}
\|f\|_{M_{\mathcal{p}, \varphi}^{\alpha, V}} & =\sup _{g \in \mathbb{H}_{n, r}>0}\left(1+\frac{r}{\rho(g)}\right)^{\alpha} \varphi(r)^{-1} r^{-Q / p}\|f\|_{L_{p}(B(g, r))} \\
\|f\|_{L M_{p, \varphi}^{\alpha, V}} & =\sup _{r>0}\left(1+\frac{r}{\rho(e)}\right)^{\alpha} \varphi(r)^{-1} r^{-Q / p}\|f\|_{L_{p}(B(e, r))}
\end{aligned}
$$

respectively. Here $e$ is the identity element in $\mathbb{H}_{n}$. Also by $W M_{p, \varphi}^{\alpha, V}=W M_{p, \varphi}^{\alpha, V}\left(\mathbb{H}_{n}\right)$ and $\operatorname{LWM}_{p, \varphi}^{\alpha, V}=$ $\operatorname{LWM} M_{p, \varphi}^{\alpha, V}\left(\mathbb{H}_{n}\right)$ we denote the weak generalized Morrey space and central weak generalized Morrey space associated with Schrödinger operator, the spaces of all functions $f \in W L_{l o c}^{p}\left(\mathbb{H}_{n}\right)$ with

$$
\begin{aligned}
\|f\|_{W M_{p, \varphi}^{\alpha, V}} & =\sup _{g \in \mathbb{H}_{n}, r>0}\left(1+\frac{r}{\rho(g)}\right)^{\alpha} \varphi(r)^{-1} r^{-Q / p}\|f\|_{W L_{p}(B(g, r))}<\infty, \\
\|f\|_{L W M_{p, \varphi}^{\alpha, Y}} & =\sup _{r>0}\left(1+\frac{r}{\rho(e)}\right)^{\alpha} \varphi(r)^{-1} r^{-Q / p}\|f\|_{W L_{p}(B(e, r))}<\infty,
\end{aligned}
$$

respectively.

\section{Remark 1.2.}

(i) When $\alpha=0$, and $\varphi(r)=r^{(\lambda-Q) / p}, M_{p, \varphi}^{\alpha, V}\left(\mathbb{R}^{\mathfrak{n}}\right)$ is the classical Morrey space $M_{p, \lambda}\left(\mathbb{R}^{\mathfrak{n}}\right)$ introduced by Morrey in [24] and $L M_{p, \varphi}^{\alpha, V}\left(\mathbb{R}^{n}\right)$ is the central Morrey space $L M_{p, \lambda}\left(\mathbb{R}^{\mathfrak{n}}\right)$ studied by Alvarez et al. in [3] on the Euclidean setting; 
(ii) when $\varphi(r)=r^{(\lambda-Q) / p}, M_{\mathcal{p}, \varphi}^{\alpha, V}\left(\mathbb{R}^{n}\right)$ is the Morrey space associated with Schrödinger operator $M_{p, \lambda}^{\alpha, V}\left(\mathbb{R}^{n}\right)$ studied by Tang and Dong in [28] on the Euclidean setting;

(iii) when $\alpha=0, M_{p, \varphi}^{\alpha, V}\left(H_{n}\right)$ is the generalized Morrey space $M_{p, \varphi}\left(\mathbb{H}_{n}\right)$ studied by Guliyev et al. in [18] and $\operatorname{LM}_{\mathrm{p}, \varphi}^{\alpha, V}\left(\mathbb{H}_{\mathrm{n}}\right)$ is the central generalized Morrey space $\operatorname{LM}_{p, \varphi}\left(\mathbb{H}_{n}\right)$ studied by Guliyev in [17], see also $[15,16,19,22]$;

(iv) $M_{\mathcal{p}, \varphi}^{\alpha, V}\left(\mathbb{R}^{n}\right)$ and $\operatorname{LM}_{\mathcal{p}, \varphi}^{\alpha, V}\left(\mathbb{R}^{n}\right)$ are the generalized Morrey space and the central generalized Morrey space associated with Schrödinger operator, respectively, studied by Guliyev in [14] on the Euclidean setting, see also $[1,2,20]$.

The classical Morrey spaces $M_{p, \lambda}\left(\mathbb{R}^{n}\right)$ were introduced by Morrey in [24] to study the local behavior of solutions to second order elliptic partial differential equations. For the properties and applications of classical Morrey spaces, we refer the readers to [7, 10, 24, 25]. The generalized Morrey spaces are defined with $r^{\lambda}$ replaced by a general non-negative function $\varphi(r)$ satisfying some assumptions (see, for example, $[8,9,18,21]$ and etc).

Definition 1.3. Let $\mathrm{L}=-\Delta_{\mathrm{H}_{n}}+\mathrm{V}$ with $\mathrm{V} \in \mathrm{RH}_{\mathrm{Q} / 2}$. The fractional integral associated with $\mathrm{L}$ is defined by

$$
\mathcal{J}_{\beta}^{\mathrm{L}} \mathrm{f}(\mathrm{g})=\mathrm{L}^{-\beta / 2} f(g)=\int_{0}^{\infty} e^{-\mathrm{tL}}(\mathrm{f})(\mathrm{g}) \mathrm{t}^{\beta / 2-1} \mathrm{dt}
$$

for $0<\beta<Q$. The commutator of $\mathcal{J}_{\beta}^{\mathrm{L}}$ is defined by

$$
\left[b, \mathcal{J}_{\beta}^{\mathrm{L}}\right] f(g)=b(g) \mathcal{J}_{\beta}^{\mathrm{L}} f(g)-\mathcal{J}_{\beta}^{\mathrm{L}}(b f)(g) .
$$

Note that, if $L=-\Delta_{\mathbb{H}_{n}}$ is the subLaplacian on $\mathbb{H}_{n}$, then $\mathcal{J}_{\beta}^{\mathrm{L}}$ and $\left[\mathrm{b}, \mathcal{J}_{\beta}^{\mathrm{L}}\right]$ are the Riesz potential $\mathrm{I}_{\beta}$ and the commutator of the Riesz potential $\left[\mathrm{b}, \mathrm{I}_{\beta}\right]$, respectively, that are

$$
I_{\beta} f(g)=\int_{\mathbb{H}_{n}} \frac{f(h)}{\left|h^{-1} g\right|^{Q-\beta}} d h, \quad\left[b, I_{\beta}\right] f(g)=\int_{\mathbb{H}_{n}} \frac{b(g)-b(h)}{\left|h^{-1} g\right|^{Q-\beta}} f(h) d h .
$$

In this paper, we consider the boundedness of the operator $\mathcal{J}_{\beta}^{\mathrm{L}}$ and its commutators $\left[\mathrm{b}, \mathrm{J}_{\beta}^{\mathrm{L}}\right]$ with $b \in \mathrm{BMO}_{\theta}\left(\mathbb{H}_{n}, \rho\right)$ on central generalized Morrey spaces $\operatorname{LM}_{\mathcal{p}, \varphi}^{\alpha, V}\left(\mathbb{H}_{n}\right)$ and generalized Morrey spaces $M_{p, \varphi}^{\alpha, V}\left(\mathbb{H}_{n}\right)$ associated with Schrödinger operator.

In this paper, we shall use the symbol $A \lesssim B$ to indicate that there exists a universal positive constant $C$, independent of all important parameters, such that $A \leqslant C B . A \approx B$ means that $A \lesssim B$ and $B \lesssim A$.

\section{Some preliminaries}

Let $\mathbb{H}_{n}$ be a Heisenberg group of dimension $2 n+1$, that is, a nilpotent Lie group with underlying manifold $\mathbb{R}^{2 n} \times \mathbb{R}$. The group structure is given by

$$
(x, t)(y, s)=\left(x+y, t+s+2 \sum_{j=1}^{n}\left(x_{n+j} y_{j}-x_{j} y_{n+j}\right)\right) .
$$

The Lie algebra of left-invariant vector fields on $\mathbb{H}_{n}$ is spanned by

$$
x_{2 n+1}=\frac{\partial}{\partial t}, x_{j}=\frac{\partial}{\partial x_{j}}+2 x_{n+j} \frac{\partial}{\partial t}, x_{n+j}=\frac{\partial}{\partial x_{n+j}}-2 x_{j} \frac{\partial}{\partial t}, j=1, \ldots, n .
$$

The non-trivial commutation relations are given by $\left[X_{j}, X_{n+j}\right]=-4 X_{2 n+1}, j=1, \ldots, n$. The sub-Laplacian $\triangle_{\mathbb{H}_{n}}$ is defined by $\Delta_{\mathbb{H}_{n}}=\sum_{j=1}^{2 n} X_{j}^{2}$. The Haar measure on $\mathbb{H}_{n}$ is simply the Lebesgue measure on $\mathbb{R}^{2 n} \times \mathbb{R}$. 
The measure of any measurable set $E \subset \mathbb{H}_{n}$ is denoted by $|E|$. The homogeneous norm on $\mathbb{H}_{n}$ is defined by

$$
|g|=\left(|x|^{4}+|t|^{2}\right)^{\frac{1}{4}}, g=(x, t) \in \mathbb{H}_{n}
$$

which leads to a left-invariant distance $d(g, h)=\left|g^{-1} h\right|$ on $\mathbb{H}_{n}$. The dilations on $\mathbb{H}_{n}$ have the form $\delta_{r}(x, t)=\left(r x, r^{2} t\right), r>0$. The Haar measure on this group coincides with the Lebesgue measure $\mathrm{dx}=\mathrm{d} x_{1} \ldots \mathrm{d} x_{2 n} \mathrm{dt}$. The identity element in $\mathbb{H}_{\mathrm{n}}$ is $e=0 \in \mathbb{R}^{2 \mathrm{n}+1}$, while the element $\mathrm{g}^{-1}$ inverse to $g=(x, t)$ is $(-x,-t)$. The ball of radius $r$ and centered at $g$ is $B(g, r)=\left\{h \in \mathbb{H}_{n}:\left|g^{-1} h\right|<r\right\}$ and $|B(g, r)|=r Q|B(0,1)|$, where $Q=2 n+2$ is the homogeneous dimension of $\mathbb{H}_{n}$. If $B=B(g, r)$, then $\lambda B$ denotes $B(g, \lambda r)$ for $\lambda>0$. Clearly, we have $|\lambda B|=\lambda Q|B|$.

For background on the analysis on the Heisenberg groups we refer the reader to [12,27].

We would like to recall the important properties concerning the critical function.

Lemma 2.1 ([23]). Let $\mathrm{V} \in \mathrm{RH}_{\mathrm{Q} / 2}$. For the associated function $\rho$ there exist $\mathrm{C}$ and $\mathrm{k}_{0} \geqslant 1$ such that

$$
C^{-1} \rho(g)\left(1+\frac{\left|h^{-1} g\right|}{\rho(g)}\right)^{-k_{0}} \leqslant \rho(h) \leqslant C \rho(g)\left(1+\frac{\left|h^{-1} g\right|}{\rho(g)}\right)^{\frac{k_{0}}{1+k_{0}}}
$$

for all $\mathrm{g}, \mathrm{h} \in \mathbb{H}_{\mathrm{n}}$.

Lemma 2.2. [2] Suppose $\mathrm{g} \in \mathrm{B}\left(\mathrm{g}_{0}, \mathrm{r}\right)$. Then for $\mathrm{k} \in \mathrm{N}$ we have

$$
\frac{1}{\left(1+\frac{2^{k} r}{\rho(g)}\right)^{N}} \lesssim \frac{1}{\left(1+\frac{2^{k} r}{\rho\left(g_{0}\right)}\right)^{N /\left(k_{0}+1\right)}}
$$

We give some inequalities about the new $\mathrm{BMO}$ space $\mathrm{BMO}_{\theta}\left(\mathbb{H}_{n}, \rho\right)$.

Lemma 2.3 ([4]). Let $1 \leqslant s<\infty$. If $\mathrm{b} \in \mathrm{BMO}_{\theta}\left(\mathbb{H}_{n}, \rho\right)$, then

$$
\left(\frac{1}{|\mathrm{~B}|} \int_{\mathrm{B}}\left|\mathrm{b}(\mathrm{h})-\mathrm{b}_{\mathrm{B}}\right|^{s} \mathrm{dh}\right)^{1 / \mathrm{s}} \leqslant[\mathrm{b}]_{\theta}\left(1+\frac{\mathrm{r}}{\rho(g)}\right)^{\theta^{\prime}}
$$

for all $\mathrm{B}=\mathrm{B}(\mathrm{g}, \mathrm{r})$, with $\mathrm{g} \in \mathbb{H}_{\mathrm{n}}$ and $\mathrm{r}>0$, where $\theta^{\prime}=\left(\mathrm{k}_{0}+1\right) \theta$ and $\mathrm{k}_{0}$ is the constant appearing in (2.1).

Lemma 2.4 ([4]). Let $1 \leqslant s<\infty, b \in \mathrm{BMO}_{\theta}\left(\mathbb{H}_{n}, \rho\right)$, and $\mathrm{B}=\mathrm{B}(\mathrm{g}, \mathrm{r})$. Then

$$
\left(\frac{1}{\left|2^{k} B\right|} \int_{2^{k} B}\left|b(h)-b_{B}\right|^{s} d h\right)^{1 / s} \leqslant[b]_{\theta} k\left(1+\frac{2^{k} r}{\rho(g)}\right)^{\theta^{\prime}}
$$

for all $\mathrm{k} \in \mathbb{N}$, with $\theta^{\prime}$ as in Lemma 2.3.

Let $K_{\beta}$ be the kernel of $\mathcal{J}_{\beta}^{\mathrm{L}}$. The following result gives the estimate on the kernel $K_{\beta}(g, y)$.

Lemma 2.5 ([5]). If $\mathrm{V} \in \mathrm{RH}_{\mathrm{Q} / 2}$, then for every $\mathrm{N}$, there exists a constant $\mathrm{C}$ such that

$$
\left|K_{\beta}(g, y)\right| \leqslant \frac{C}{\left(1+\frac{\left|h^{-1} g\right|}{\rho(g)}\right)^{N}} \frac{1}{\left|h^{-1} g\right|^{Q-\beta}} .
$$

Finally, we recall a relationship between essential supremum and essential infimum.

Lemma 2.6 ([29]). Let $\mathrm{f}$ be a real-valued nonnegative function and measurable on $E$. Then

$$
\left(\underset{g \in E}{\operatorname{essinf} f(g))^{-1}}=\underset{g \in E}{\operatorname{ess} \sup } \frac{1}{f(g)} .\right.
$$


Lemma 2.7 ([20]). Let $\varphi$ be a positive measurable function on $(0, \infty), 1 \leqslant p<\infty, \alpha \geqslant 0$, and $\mathrm{V} \in \mathrm{RH}_{\mathrm{q}}, \mathrm{q} \geqslant 1$. If

$$
\sup _{t<r<\infty}\left(1+\frac{r}{\rho(e)}\right)^{\alpha} \frac{r^{-\frac{n}{p}}}{\varphi(r)}=\infty \quad \text { for some } t>0,
$$

then $\operatorname{LM}_{\mathcal{p}, \varphi}^{\alpha, V}\left(\mathbb{H}_{n}\right)=\Theta$, where $\Theta$ is the set of all functions equivalent to 0 on $\mathbb{H}_{n}$.

Lemma 2.8 ([2,8]). Let $\varphi$ be a positive measurable function on $(0, \infty), 1 \leqslant p<\infty, \alpha \geqslant 0$, and $\mathrm{V} \in \mathrm{RH}_{\mathrm{q}}, \mathrm{q} \geqslant 1$.

(i) If

$$
\sup _{\mathrm{t}<\mathrm{r}<\infty}\left(1+\frac{\mathrm{r}}{\rho(\mathrm{g})}\right)^{\alpha} \frac{\mathrm{r}^{-\frac{\mathrm{Q}}{\mathrm{p}}}}{\varphi(\mathrm{r})}=\infty \quad \text { for some } \mathrm{t}>0 \text { and for all } \mathrm{g} \in \mathbb{H}_{\mathrm{n}}
$$

then $\mathrm{M}_{\mathrm{p}, \varphi}^{\alpha, \mathrm{V}}\left(\mathbb{H}_{\mathrm{n}}\right)=\Theta$.

(ii) If

$$
\sup _{0<r<\tau}\left(1+\frac{r}{\rho(g)}\right)^{\alpha} \varphi(r)^{-1}=\infty \quad \text { for some } \tau>0 \text { and for all } \mathrm{g} \in \mathbb{H}_{\mathrm{n}}
$$

then $M_{p, \varphi}^{\alpha, V}\left(\mathbb{H}_{n}\right)=\Theta$.

Remark 2.9. We denote by $\Omega_{p, l o c}^{\alpha, V}$ (see [20]) the sets of all positive measurable functions $\varphi$ on $(0, \infty)$ such that for all $t>0$,

$$
\left\|\left(1+\frac{r}{\rho(e)}\right)^{\alpha} \frac{r^{-\frac{n}{p}}}{\varphi(r)}\right\|_{L_{\infty}(t, \infty)}<\infty
$$

Moreover, we denote by $\Omega_{\mathrm{p}}^{\alpha, V}$ (see $[2,8]$ ) the sets of all positive measurable functions $\varphi$ on $(0, \infty)$ such that for all $t>0$,

$$
\sup _{g \in \mathbb{H}_{n}}\left\|\left(1+\frac{r}{\rho(g)}\right)^{\alpha} \frac{r^{-\frac{Q}{p}}}{\varphi(r)}\right\|_{L_{\infty}(t, \infty)}<\infty, \quad \text { and } \sup _{g \in \mathbb{H}_{n}}\left\|\left(1+\frac{r}{\rho(g)}\right)^{\alpha} \varphi(r)^{-1}\right\|_{L_{\infty}(0, t)}<\infty,
$$

respectively.

For the non-triviality of the spaces $\operatorname{LM}_{\mathbf{p}, \varphi}^{\alpha, V}\left(\mathbb{H}_{n}\right), M_{p, \varphi}^{\alpha, V}\left(\mathbb{H}_{n}\right)$ we always assume that $\varphi \in \Omega_{p^{\prime}, l^{\prime}}^{\alpha, V} \varphi \in$ $\Omega_{\mathrm{p}}^{\alpha, V}$, respectively.

\section{Main results}

We first prove the following local Guliyev estimates (see $[13,15,17])$ for the operator $\mathcal{J}_{\beta}^{\mathrm{L}}$.

Theorem 3.1. Let $\mathrm{V} \in \mathrm{RH}_{\mathrm{Q} / 2}$. If $1<\mathrm{p}<\mathrm{Q} / \beta, 1 / \mathrm{q}=1 / \mathrm{p}-\beta / \mathrm{Q}$, then the inequality

$$
\left\|\mathcal{J}_{\beta}^{L}(f)\right\|_{L_{q}\left(B\left(g_{0}, r\right)\right)} \lesssim r^{\frac{Q}{q}} \int_{2 r}^{\infty} \frac{\|f\|_{L_{p}\left(B\left(g_{0}, t\right)\right)}}{t^{\frac{Q}{q}}} \frac{d t}{t}
$$

holds for any $\mathrm{f} \in \mathrm{L}_{\mathrm{loc}}^{\mathrm{p}}\left(\mathrm{H}_{\mathrm{n}}\right)$. Moreover, for $\mathrm{p}=1$ the inequality

$$
\left\|\mathcal{J}_{\beta}^{\mathrm{L}}(\mathrm{f})\right\|_{W \mathrm{~W}_{\mathrm{Q}-\beta}}\left(\mathrm{B}\left(\mathrm{g}_{0}, \mathrm{r}\right)\right) \lesssim \mathrm{r}^{\mathrm{Q}-\beta} \int_{2 \mathrm{r}}^{\infty} \frac{\|f\|_{\mathrm{L}_{1}\left(\mathrm{~B}\left(\mathrm{~g}_{0}, \mathrm{t}\right)\right)}}{\mathrm{t}^{\mathrm{Q}-\beta}} \frac{\mathrm{dt}}{\mathrm{t}}
$$

holds for any $f \in \mathrm{L}_{\text {loc }}^{1}\left(\mathrm{H}_{n}\right)$. 
Proof. For arbitrary $g_{0} \in \mathbb{H}_{n}$, set $B=B\left(g_{0}, r\right)$ and $\lambda B=B\left(g_{0}, \lambda r\right)$ for any $\lambda>0$. We write $f$ as $f=f_{1}+f_{2}$, where $f_{1}(h)=f(h) \chi_{B\left(g_{0}, 2 r\right)}(h)$, and $\chi_{B\left(g_{0}, 2 r\right)}$ denotes the characteristic function of $B\left(g_{0}, 2 r\right)$. Then

$$
\left\|\mathcal{J}_{\beta}^{\mathrm{L}}(\mathrm{f})\right\|_{\mathrm{L}_{q}\left(B\left(g_{0}, r\right)\right)} \leqslant\left\|\mathcal{J}_{\beta}^{\mathrm{L}}\left(f_{1}\right)\right\|_{L_{q}\left(B\left(g_{0}, r\right)\right)}+\left\|\mathcal{J}_{\beta}^{L}\left(f_{2}\right)\right\|_{L_{q}\left(B\left(g_{0}, r\right)\right)} .
$$

Since $f_{1} \in L_{p}\left(\mathbb{R}^{n}\right)$ and from the boundedness of $\mathcal{J}_{\beta}^{L}$ from $L_{p}\left(\mathbb{H}_{n}\right)$ to $L_{q}\left(\mathbb{H}_{n}\right)$ (see $\left.[23,27]\right)$ it follows that

$$
\left\|\mathcal{J}_{\beta}^{L}\left(f_{1}\right)\right\|_{L_{q}\left(B\left(g_{0}, r\right)\right)} \lesssim\|f\|_{L_{p}\left(B\left(g_{0}, 2 r\right)\right)} \lesssim r^{\frac{Q}{q}}\|f\|_{L_{p}\left(B\left(g_{0}, 2 r\right)\right)} \int_{2 r}^{\infty} \frac{d t}{t^{\frac{Q}{q}+1}} \lesssim r^{\frac{Q}{q}} \int_{2 r}^{\infty} \frac{\|f\|_{L_{p}\left(B\left(g_{0}, t\right)\right)}}{t^{\frac{Q}{q}}} \frac{d t}{t} .
$$

To estimate $\left\|\mathcal{J}_{\beta}^{\mathrm{L}}\left(\mathrm{f}_{2}\right)\right\|_{\mathrm{L}_{p}\left(\mathrm{~B}\left(\mathrm{~g}_{0}, \mathrm{r}\right)\right)}$, obverse that $\mathrm{g} \in \mathrm{B}, \mathrm{h} \in(2 \mathrm{~B})^{\mathrm{c}}$ implies $\left|\mathrm{h}^{-1} \mathrm{~g}\right| \approx\left|\mathrm{h}^{-1} \mathrm{~g}_{0}\right|$. Then by (2.2) we have

$$
\sup _{g \in B}\left|\mathcal{J}_{\beta}^{L}\left(f_{2}\right)(g)\right| \leqslant \int_{(2 B)^{c}}\left|K_{\beta}(g, h) f(h)\right| d h \lesssim \int_{(2 B)^{c}} \frac{|f(h)|}{\left|h^{-1} g_{0}\right|^{Q-\beta}} d y \lesssim \sum_{k=1}^{\infty}\left(2^{k+1} r\right)^{-Q+\beta} \int_{2^{k+1} B}|f(h)| d h .
$$

By Hölder's inequality we get

$$
\begin{aligned}
\sup _{g \in B}\left|J_{\beta}^{L}\left(f_{2}\right)(g)\right| & \lesssim \sum_{k=1}^{\infty}\|f\|_{L_{p}\left(2^{k+1} B\right)}\left(2^{k+1} r\right)^{-1-\frac{Q}{p}+\beta} \int_{2^{k} r}^{2^{k+1} r} d t \\
& \lesssim \sum_{k=1}^{\infty} \int_{2^{k} r}^{2^{k+1} r} \frac{\|f\|_{L_{p}\left(B\left(g_{0}, t\right)\right)}}{t^{\frac{Q}{q}}} \frac{d t}{t} \lesssim \int_{2 r}^{\infty} \frac{\|f\|_{L_{p}\left(B\left(g_{0}, t\right)\right)}}{t^{\frac{Q}{q}}} \frac{d t}{t} .
\end{aligned}
$$

Then

$$
\left\|\mathcal{J}_{\beta}^{L}\left(f_{2}\right)\right\|_{L_{q}\left(B\left(g_{0}, r\right)\right)} \lesssim r \frac{Q}{q} \int_{2 r}^{\infty} \frac{\|f\|_{L_{p}\left(B\left(g_{0}, t\right)\right)}}{t^{\frac{Q}{q}}} \frac{d t}{t}
$$

holds for $1 \leqslant p<Q / \beta$. Therefore, by (3.1) and (3.3) we get

$$
\left\|\mathcal{J}_{\beta}^{\mathrm{L}}(f)\right\|_{L_{q}\left(B\left(g_{0}, r\right)\right)} \lesssim r^{\frac{Q}{q}} \int_{2 r}^{\infty} \frac{\|f\|_{L_{p}\left(B\left(g_{0}, t\right)\right)}}{t^{\frac{Q}{q}}} \frac{d t}{t}
$$

holds for $1 \leqslant p<Q / \beta$.

When $p=1$, by the boundedness of $\mathcal{J}_{\beta}^{L}$ from $L_{1}\left(\mathbb{H}_{n}\right)$ to $W L_{\frac{Q}{Q-\beta}}\left(H_{n}\right)$ (see $\left.[23,27]\right)$, we get

$$
\left\|\mathcal{J}_{\beta}^{\mathrm{L}}\left(\mathrm{f}_{1}\right)\right\|_{W \mathrm{~L}_{\mathrm{Q}} \mathrm{Q}-\mathrm{B}}\left(\mathrm{B}\left(\mathrm{g}_{0}, \mathrm{r}\right)\right) \lesssim\|f\|_{\mathrm{L}_{1}\left(\mathrm{~B}\left(\mathrm{~g}_{0}, 2 \mathrm{r}\right)\right)} \lesssim \mathrm{r}^{\mathrm{Q}-\beta} \int_{2 \mathrm{r}}^{\infty} \frac{\|\mathrm{f}\|_{\mathrm{L}_{1}\left(\mathrm{~B}\left(\mathrm{~g}_{0}, \mathrm{t}\right)\right)}}{\mathrm{t}^{\mathrm{Q}-\beta}} \frac{\mathrm{dt}}{\mathrm{t}}
$$

By (3.3) we have

$$
\left\|\mathcal{J}_{\beta}^{\mathrm{L}}\left(\mathrm{f}_{2}\right)\right\|_{W \mathrm{~W}_{\mathrm{Q}-\beta}}\left(B\left(\mathrm{~g}_{0}, \mathrm{r}\right)\right) \leqslant\left\|\mathcal{J}_{\beta}^{\mathrm{L}}\left(\mathrm{f}_{2}\right)\right\|_{\mathrm{L}_{\mathrm{Q}-\beta}}\left(\mathrm{B}\left(\mathrm{g}_{0}, 2 \mathrm{r}\right)\right) \lesssim \mathrm{r}^{\mathrm{Q}-\beta} \int_{2 \mathrm{r}}^{\infty} \frac{\|f\|_{\mathrm{L}_{1}\left(\mathrm{~B}\left(\mathrm{~g}_{0}, \mathrm{t}\right)\right)}}{\mathrm{t} \mathrm{Q}-\beta} \frac{d t}{t} .
$$

Then

$$
\left\|J_{\beta}^{L}(f)\right\|_{W L} \frac{Q}{Q-\beta}\left(B\left(g_{0}, r\right)\right) \lesssim r Q-\beta \int_{2 r}^{\infty} \frac{\|f\|_{L_{1}\left(B\left(g_{0}, t\right)\right)}}{t^{Q-\beta}} \frac{d t}{t} .
$$

Our main results are as follows.

Theorem 3.2. Let $\mathrm{V} \in \mathrm{RH}_{\mathrm{Q} / 2}, \alpha \geqslant 0,1<\mathrm{p}<\mathrm{Q} / \beta, 1 / \mathrm{q}=1 / \mathrm{p}-\beta / \mathrm{Q}$ and $\varphi_{1} \in \Omega_{\mathrm{p}, \mathrm{loc}^{\prime}}^{\alpha, \varphi_{2}} \in \Omega_{\mathrm{q}, \mathrm{loc}}^{\alpha, \mathrm{V}}$ satisfy the condition

$$
\int_{\mathrm{r}}^{\infty} \frac{\operatorname{ess} \inf \varphi_{1}(s) s^{\frac{Q}{p}}}{t^{\frac{Q}{q}}} \frac{d t}{t} \leqslant c_{0} \varphi_{2}(r)
$$

where $\mathrm{c}_{0}$ does not depend on $\mathrm{r}$. Then the operator $\mathcal{J}_{\beta}^{\mathrm{L}}$ is bounded on $\mathrm{LM}_{\mathbf{p}_{,}, \varphi_{1}}^{\alpha, \mathrm{V}}\left(\mathbb{H}_{\mathfrak{n}}\right)$ to $\mathrm{LM}_{\mathbf{q}, \varphi_{2}}^{\alpha, V}\left(\mathbb{H}_{\mathfrak{n}}\right)$ for $p>1$ and 
from $\operatorname{LM}_{1, \varphi_{1}}^{\alpha, V_{1}}\left(\mathbb{H}_{n}\right)$ to $\operatorname{LWM}_{\frac{\mathrm{Q}}{\mathrm{Q}-\beta}, \varphi_{2}}^{\alpha, \mathrm{V}}\left(\mathbb{H}_{\mathrm{n}}\right)$.

Proof. From Lemma 2.6, we have

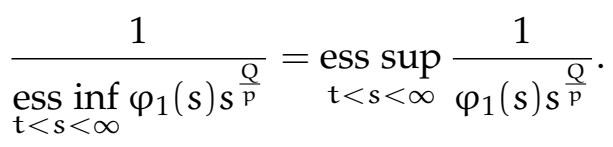

Note the fact that $\|f\|_{L_{p}(B(e, r))}$ is a nondecreasing function of $r$, and $f \in L M_{p_{,}, \varphi_{1}}^{\alpha, V}\left(H_{n}\right)$, then

$$
\begin{aligned}
\frac{\left(1+\frac{r}{\rho(e)}\right)^{\alpha}\|f\|_{L_{p}(B(e, r))}}{\operatorname{essinf}_{r<s<\infty} \varphi_{1}(s) s^{\frac{Q}{p}}} & \lesssim \operatorname{ess~sup~}_{r<s<\infty} \frac{\left(1+\frac{r}{\rho(e)}\right)^{\alpha}\|f\|_{L_{p}(B(e, r))}}{\varphi_{1}(s) s^{\frac{Q}{p}}} \\
& \lesssim \sup _{0<s<\infty} \frac{\left(1+\frac{s}{\rho(e)}\right)^{\alpha}\|f\|_{L_{p}(B(e, s))}}{\varphi_{1}(s) s^{\frac{Q}{p}}} \lesssim\|f\|_{L_{M_{p}, \varphi_{1}}^{\alpha, V}} .
\end{aligned}
$$

Since $\alpha \geqslant 0$, and $\left(\varphi_{1}, \varphi_{2}\right)$ satisfies the condition (3.4), then

$$
\begin{aligned}
& \int_{2 r}^{\infty} \frac{\|f\|_{L_{p}(B(e, \tau))}}{\tau^{\frac{Q}{q}}} \frac{d \tau}{\tau}=\int_{2 r}^{\infty} \frac{\left(1+\frac{\tau}{\rho(e)}\right)^{\alpha}\|f\|_{L_{p}(B(e, \tau))}}{\underset{\tau<s}{\operatorname{ess} \inf \inf _{\tau<\infty} \varphi_{1}(s) s^{\frac{Q}{p}}}} \frac{(s) s^{\frac{Q}{p}}}{\left(1+\frac{\tau}{\rho(e)}\right)^{\alpha} \tau^{\frac{Q}{q}}} \frac{d \tau}{\tau} \\
& \lesssim\|f\|_{L_{M_{p}, \varphi_{1}}^{\alpha, V}} \int_{2 r}^{\infty} \frac{\underset{\tau<s<\infty}{\operatorname{essinf}} \varphi_{1}(s) s^{\frac{Q}{p}}}{\left(1+\frac{\tau}{\rho(e)}\right)^{\alpha} \tau^{\frac{Q}{q}}} \frac{d \tau}{\tau} \\
& \lesssim\|f\|_{L_{M_{\mathcal{P}, \varphi_{1}}}^{\alpha, V}}\left(1+\frac{r}{\rho(e)}\right)^{-\alpha} \\
& \times \int_{r}^{\infty} \frac{\underset{\tau<s<\infty}{\operatorname{essinf}} \varphi_{1}(s) s^{\frac{Q}{p}}}{\tau^{\frac{Q}{q}}} \frac{d \tau}{\tau} \lesssim\|f\|_{L_{M_{\mathcal{P}, \varphi_{1}}}^{\alpha, V}}\left(1+\frac{\mathrm{r}}{\rho(e)}\right)^{-\alpha} \varphi_{2}(\mathrm{r}) .
\end{aligned}
$$

Then by Theorem 3.1 we get

$$
\begin{aligned}
& \left\|\mathcal{J}_{\beta}^{\mathrm{L}}(\mathrm{f})\right\|_{\mathrm{LM}_{\mathrm{q}, \varphi_{2}}^{\alpha}, \nu} \lesssim \sup _{\mathrm{r}>0}\left(1+\frac{\mathrm{r}}{\rho(e)}\right)^{\alpha} \varphi_{2}(\mathrm{r})^{-1} \mathrm{r}^{-\mathrm{Q} / \mathrm{q}}\left\|\mathcal{J}_{\beta}^{\mathrm{L}}(\mathrm{f})\right\|_{\mathrm{L}_{\mathrm{p}}(\mathrm{B}(e, r))} \\
& \lesssim \sup _{r>0}\left(1+\frac{r}{\rho(e)}\right)^{\alpha} \varphi_{2}(r)^{-1} \int_{2 r}^{\infty} \frac{\|f\|_{L_{p}(B(e, t))}}{t^{\frac{Q}{q}}} \frac{d t}{t} \lesssim\|f\|_{L M_{p^{\prime}, \varphi_{1}}^{\alpha, V}} .
\end{aligned}
$$

Let $q=\frac{Q}{Q-\beta}$, similar to the estimates of (3.5) we have

$$
\int_{2 r}^{\infty} \frac{\|f\|_{L_{1}(B(e, \tau))}}{\tau \tau^{Q-\beta}} \frac{d \tau}{\tau} \lesssim\|f\|_{L_{1, \varphi_{1}}^{\alpha, V}}\left(1+\frac{r}{\rho(e)}\right)^{-\alpha} \varphi_{2}(r) .
$$

Thus by Theorem 3.1 we get

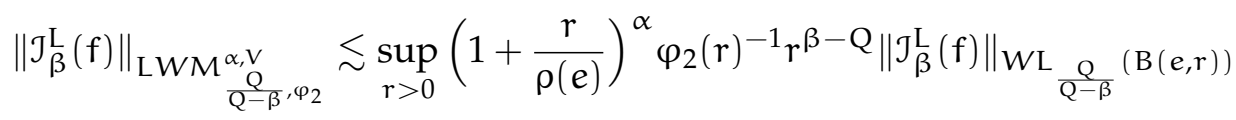

$$
\begin{aligned}
& \lesssim \sup _{\mathrm{r}>0}\left(1+\frac{\mathrm{r}}{\rho(e)}\right)^{\alpha} \varphi_{2}(\mathrm{r})^{-1} \int_{2 \mathrm{r}}^{\infty} \frac{\|\mathrm{f}\|_{\mathrm{L}_{1}(\mathrm{~B}(e, \tau))}}{\tau \mathrm{Q}-\beta} \frac{\mathrm{d} \tau}{\tau} \lesssim\|\mathrm{f}\|_{\mathrm{LM}_{1, \varphi_{1}}^{\alpha, V}} .
\end{aligned}
$$

Corollary 3.3. Let $\mathrm{V} \in \mathrm{RH}_{\mathrm{Q} / 2}, \alpha \geqslant 0,1<\mathrm{p}<\mathrm{Q} / \beta, 1 / \mathrm{q}=1 / \mathrm{p}-\beta / \mathrm{Q}$ and $\varphi_{1} \in \Omega_{\mathrm{p}}^{\alpha, \mathrm{V}}, \varphi_{2} \in \Omega_{\mathrm{q}}^{\alpha, \mathrm{V}}$ satisfy the condition (3.4). Then the operator $\mathcal{J}_{\beta}^{L}$ is bounded on $M_{p_{,}, \varphi_{1}}^{\alpha, V}\left(\mathbb{H}_{n}\right)$ to $M_{q_{,} \varphi_{2}}^{\alpha, V}\left(\mathbb{H}_{n}\right)$ for $p>1$ and from $M_{1, \varphi_{1}}^{\alpha, V}\left(\mathbb{H}_{n}\right)$ to $W M_{\frac{\mathrm{Q}}{\mathrm{Q}-\beta}, \varphi_{2}}^{\alpha, \mathrm{V}}\left(\mathbb{H}_{\mathrm{n}}\right)$. 
Theorem 3.4. Let $\mathrm{V} \in \mathrm{RH}_{\mathrm{Q} / 2}, \alpha \geqslant 0,1<\mathrm{p}<\mathrm{Q} / \beta, 1 / \mathrm{q}=1 / \mathrm{p}-\beta / \mathrm{Q}$ and $\varphi_{1} \in \Omega_{\mathrm{p}, \text { loc }}^{\alpha, \mathrm{V}} \varphi_{2} \in \Omega_{\mathrm{q}, \text { loc }}^{\alpha, \mathrm{V}}$ satisfy the condition

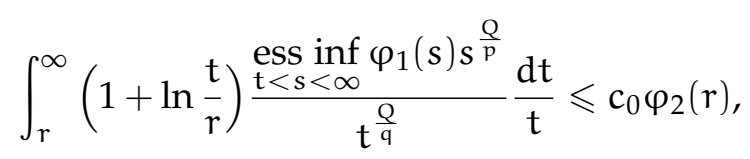

where $\mathrm{c}_{0}$ does not depend on $\mathrm{r}$. If $\mathrm{b} \in \mathrm{BMO}_{\theta}\left(\mathbb{H}_{\mathrm{n}}, \rho\right)$, then the operator $\left[\mathrm{b}, \mathcal{J} \mathrm{J}_{\beta}\right]$ is bounded from $\mathrm{LM}_{\mathrm{p}^{\prime}, \varphi_{1}}^{\alpha}\left(\mathbb{H}_{\mathfrak{n}}\right)$ to $\operatorname{LM}_{q, \varphi_{2}}^{\alpha, V}\left(\mathbb{H}_{n}\right)$.

Corollary 3.5. Let $\mathrm{V} \in \mathrm{RH}_{\mathrm{Q} / 2}, \alpha \geqslant 0,1<\mathrm{p}<\mathrm{Q} / \beta, 1 / \mathrm{q}=1 / \mathrm{p}-\beta / \mathrm{Q}$ and $\varphi_{1} \in \Omega_{\mathrm{p}}^{\alpha, \mathrm{V}}, \varphi_{2} \in \Omega_{\mathrm{q}}^{\alpha, \mathrm{V}}$ satisfy the condition (3.6). If $\mathrm{b} \in \mathrm{BMO}_{\theta}\left(\mathbb{H}_{n}, \rho\right)$, then the operator $\left[\mathrm{b}, \mathcal{J}_{\beta}^{\mathrm{L}}\right]$ is bounded from $\mathrm{M}_{\mathrm{p}^{\prime}, \varphi_{1}}^{\alpha, V_{1}}\left(\mathbb{H}_{n}\right)$ to $\mathrm{M}_{\mathrm{q}, \varphi_{2}}^{\alpha, V_{2}}\left(\mathbb{H}_{n}\right)$.

As the proof of Theorem 3.4, it suffices to prove the following local Guliyev estimates (see [16]) for the commutator operator $[b, \mathcal{J} \mathrm{L}]$.

Theorem 3.6. Let $\mathrm{V} \in \mathrm{RH}_{\mathrm{Q} / 2}, \mathrm{~b} \in \mathrm{BMO}_{\theta}\left(\mathbb{H}_{n}, \rho\right)$. If $1<\mathrm{p}<\mathrm{Q} / \beta, 1 / \mathrm{q}=1 / \mathrm{p}-\beta / \mathrm{Q}$ then the inequality

$$
\left\|\left[b, \mathcal{J}_{\beta}^{\mathrm{L}}\right](\mathrm{f})\right\|_{L_{q}\left(B\left(g_{0}, r\right)\right)} \lesssim[b]_{\theta} r^{\frac{Q}{q}} \int_{2 r}^{\infty}\left(1+\ln \frac{\tau}{r}\right) \frac{\|f\|_{L_{p}\left(B\left(g_{0}, \tau\right)\right)}}{\tau^{\frac{Q}{q}}} \frac{d \tau}{\tau}
$$

holds for any $f \in \mathrm{L}_{\mathrm{loc}}^{\mathrm{p}}\left(\mathrm{H}_{\mathrm{n}}\right)$.

Proof. We write $f$ as $f=f_{1}+f_{2}$, where $f_{1}(h)=f(h) \chi_{B\left(g_{0}, 2 r\right)}(h)$. Then

$$
\left\|\left[b, J_{\beta}^{L}\right](f)\right\|_{L_{q}\left(B\left(g_{0}, r\right)\right)} \leqslant\left\|\left[b, J_{\beta}^{L}\right]\left(f_{1}\right)\right\|_{L_{q}\left(B\left(g_{0}, r\right)\right)}+\left\|\left[b, J_{\beta}^{L}\right]\left(f_{2}\right)\right\|_{L_{q}\left(B\left(g_{0}, r\right)\right)} .
$$

By the boundedness of $\left[b, \mathcal{J}_{\beta}^{L}\right]$ on $L_{p}\left(\mathbb{H}_{n}\right)$ to $L_{q}\left(\mathbb{H}_{n}\right)$ (see $\left.[23,27]\right)$ and $(3.1)$ we get

$$
\begin{aligned}
\left\|\left[b, J_{\beta}^{L}\right]\left(f_{1}\right)\right\|_{L_{q}\left(B\left(g_{0}, r\right)\right)} \lesssim[b]_{\theta}\|f\|_{L_{p}\left(B\left(g_{0}, 2 r\right)\right)} & \lesssim[b]_{\theta} r^{\frac{Q}{q}} \int_{2 r}^{\infty} \frac{\|f\|_{L_{p}\left(B\left(g_{0}, \tau\right)\right)}}{\tau^{\frac{Q}{q}}} \frac{d \tau}{\tau} \\
& \lesssim[b]_{\theta} r^{\frac{Q}{q}} \int_{2 r}^{\infty}\left(1+\ln \frac{\tau}{r}\right) \frac{\|f\|_{L_{p}\left(B\left(g_{0}, \tau\right)\right)}}{\tau^{\frac{Q}{q}}} \frac{d \tau}{\tau} .
\end{aligned}
$$

We now turn to deal with the term $\left\|\left[b, J_{\beta}^{L}\right]\left(f_{2}\right)\right\|_{L_{q}\left(B\left(g_{0}, r\right)\right)}$. For any given $g \in B\left(g_{0}, r\right)$ we have

$$
\left|\left[b, J_{\beta}^{L}\right] f_{2}(g)\right| \leqslant\left|b(g)-b_{2 B}\right|\left|J_{\beta}^{L}\left(f_{2}\right)(g)\right|+\left|J_{\beta}^{L}\left(\left(b-b_{2 B}\right) f_{2}\right)(g)\right| .
$$

Then by (3.2), Lemma 2.3, and taking $N \geqslant\left(k_{0}+1\right) \theta$ we get

$$
\begin{aligned}
\left\|\left(b(g)-b_{2 B}\right) \mathcal{J}_{\beta}^{L}\left(f_{2}\right)\right\|_{L_{q}\left(B\left(g_{0}, r\right)\right)} & \lesssim[b]_{\theta} r^{\frac{Q}{q}}\left(1+\frac{2 r}{\rho\left(g_{0}\right)}\right)^{\theta-N /\left(k_{0}+1\right)} \int_{2 r}^{\infty} \frac{\|f\|_{L_{p}\left(B\left(g_{0}, \tau\right)\right)}}{\tau^{\frac{Q}{q}}} \frac{d \tau}{\tau} \\
& \lesssim[b]_{\theta} r^{\frac{Q}{q}} \int_{2 r}^{\infty}\left(1+\ln \frac{\tau}{r}\right) \frac{\|f\|_{L_{p}\left(B\left(g_{0}, \tau\right)\right)}}{\tau^{\frac{Q}{q}}} \frac{d \tau}{\tau} .
\end{aligned}
$$

Finally, let us estimate $\left\|\mathcal{J}_{\beta}^{L}\left(\left(b-b_{2 B}\right) f_{2}\right)\right\|_{L_{q}\left(B\left(g_{0}, r\right)\right)}$. By (2.2), Lemma 2.2, and (3.2) we have

$$
\begin{aligned}
\sup _{g \in B}\left|\mathcal{J}_{\beta}\left(\left(b-b_{2 B}\right) f_{2}\right)(g)\right| & \lesssim \int_{(2 B)^{c}} \frac{1}{\left(1+\frac{\left|h^{-1} g\right|}{\rho(g)}\right)^{N}} \frac{\left|b(h)-b_{2 B}\right||f(h)|}{\left|h^{-1} g_{0}\right|^{Q-\beta}} d h \\
& \lesssim \sum_{k=1}^{\infty} \frac{1}{\left(2^{k} r\right) Q-\beta\left(1+\frac{2^{k} r}{\rho(g)}\right)^{N}} \int_{2^{k+1} B}\left|b(h)-b_{2 B} \| f(h)\right| d h
\end{aligned}
$$




$$
\lesssim \sum_{k=1}^{\infty} \frac{1}{\left(2^{k} r\right) Q-\beta\left(1+\frac{2^{k} r}{\rho\left(g_{0}\right)}\right)^{N /\left(k_{0}+1\right)}} \int_{2^{k+1} B}\left|b(h)-b_{2 B} \| f(h)\right| d h .
$$

Note that

$$
\begin{aligned}
\int_{2^{k+1} B}\left|b(h)-b_{2 B} \| f(h)\right| d h & \lesssim\left(\int_{2^{k+1} B}\left|b(h)-b_{2 B}\right|^{p^{\prime}}\right)^{1 / p^{\prime}}\|f\|_{L_{p}\left(B\left(g_{0}, 2^{k+1} r\right)\right)} \\
& \lesssim[b]_{\theta} k\left(1+\frac{2^{k} r}{\rho\left(g_{0}\right)}\right)^{\theta^{\prime}}\left(2^{k} r\right)^{\frac{Q}{p^{\prime}}}\|f\|_{L_{p}\left(B\left(g_{0}, 2^{k+1} r\right)\right)} .
\end{aligned}
$$

Then

$$
\begin{aligned}
\sup _{g \in B}\left|\mathcal{J}_{\beta}^{L}\left(\left(b-b_{2 B}\right) f_{2}\right)(g)\right| & \lesssim[b]_{\theta} \sum_{k=1}^{\infty} \frac{k\left(2^{k} r\right)^{-\frac{Q}{p}+\beta}}{\left(1+\frac{2^{k} r}{\rho\left(g_{0}\right)}\right)^{N /\left(k_{0}+1\right)-\theta^{\prime}}}\|f\|_{L_{p}\left(B\left(g_{0}, 2^{k+1} r\right)\right)} \\
& \lesssim[b]_{\theta} \sum_{k=1}^{\infty} k\left(2^{k} r\right)^{-\frac{Q}{q}}\|f\|_{L_{p}\left(B\left(g_{0}, 2^{k+1} r\right)\right)} \lesssim[b]_{\theta} \sum_{k=1}^{\infty} k \int_{2^{k} r}^{2^{k+1} r} \frac{\|f\|_{L_{p}\left(B\left(g_{0}, \tau\right)\right)}}{\tau^{\frac{Q}{q}}} \frac{d \tau}{\tau} .
\end{aligned}
$$

Since $2^{k} r \leqslant t \leqslant 2^{k+1} r$, then $k \approx \ln \frac{\tau}{r}$. Thus

$$
\begin{aligned}
\sup _{g \in B}\left|J_{\beta}^{L}\left(\left(b-b_{2 B}\right) f_{2}\right)(g)\right| & \lesssim[b]_{\theta} \sum_{k=1}^{\infty} k \int_{2^{k} r}^{2^{k+1} r} \frac{\|f\|_{L_{p}\left(B\left(g_{0}, \tau\right)\right)}}{\tau^{\frac{Q}{q}}} \frac{d \tau}{\tau} \\
& \lesssim[b]_{\theta} \sum_{k=1}^{\infty} \int_{2^{k} r}^{2^{k+1} r} \ln \frac{\tau}{r} \frac{\|f\|_{L_{p}\left(B\left(g_{0}, \tau\right)\right)}}{\tau^{\frac{Q}{q}}} \frac{d \tau}{\tau} \lesssim[b]_{\theta} \int_{2 r}^{\infty}\left(1+\ln \frac{\tau}{r}\right) \frac{\|f\|_{L_{p}\left(B\left(g_{0}, \tau\right)\right)}}{\tau^{\frac{Q}{q}}} \frac{d \tau}{\tau} .
\end{aligned}
$$

Then

$$
\left\|\mathcal{J}_{\beta}^{L}\left(\left(b-b_{2 B}\right) f_{2}\right)\right\|_{L_{q}\left(B\left(g_{0}, r\right)\right)} \lesssim[b]_{\theta} r^{\frac{Q}{q}} \int_{2 r}^{\infty}\left(1+\ln \frac{\tau}{r}\right) \frac{\|f\|_{L_{p}\left(B\left(g_{0}, \tau\right)\right)}}{\tau^{\frac{Q}{q}}} \frac{d \tau}{\tau} .
$$

Combining (3.7), (3.8), and (3.9), the proof of Theorem 3.6 is completed.

Proof of Theorem 3.4. Since $f \in \mathrm{LM}_{\mathrm{p}, \varphi_{1}}^{\alpha, V_{1}}\left(\mathbb{H}_{n}\right)$ and $\left(\varphi_{1}, \varphi_{2}\right)$ satisfies the condition (3.6), by (3.5) we have

$$
\begin{aligned}
& \int_{2 r}^{\infty}\left(1+\ln \frac{\tau}{r}\right) \frac{\|f\|_{L_{p}(B(e, \tau))}}{\tau^{\frac{Q}{q}}} \frac{d \tau}{\tau}=\int_{2 r}^{\infty} \frac{\left(1+\frac{\tau}{\rho(e)}\right)^{\alpha}\|f\|_{L_{p}(B(e, \tau))}}{\operatorname{essinf}_{\tau<s<\infty} \varphi_{1}(s) s^{\frac{Q}{p}}}\left(1+\ln \frac{\tau}{r}\right) \frac{\underset{\tau<s<\infty}{\operatorname{essinf}} \varphi_{1}(s) s^{\frac{Q}{p}}}{\left(1+\frac{\tau}{\rho(e)}\right)^{\alpha} \tau^{\frac{Q}{q}}} \frac{d \tau}{\tau}
\end{aligned}
$$

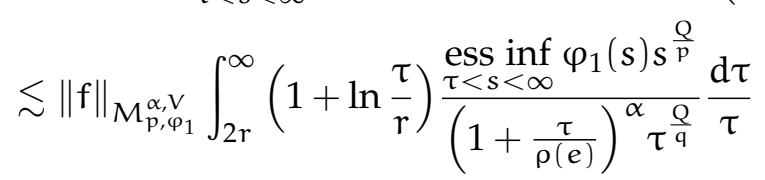

$$
\begin{aligned}
& \lesssim\|f\|_{M_{p, \varphi_{1}}^{\alpha, v}}\left(1+\frac{r}{\rho(e)}\right)^{-\alpha} \int_{r}^{\infty}\left(1+\ln \frac{\tau}{r}\right) \frac{\underset{\tau}{\operatorname{ess} \inf <\infty} \varphi_{1}(s) s^{\frac{Q}{p}}}{\tau^{\frac{Q}{q}}} \frac{d \tau}{\tau} \\
& \lesssim\|f\|_{M_{p, \varphi_{1}}^{\alpha, V}}\left(1+\frac{r}{\rho(e)}\right)^{-\alpha} \varphi_{2}(r) .
\end{aligned}
$$

Then from Theorem 3.6 we get

$$
\begin{aligned}
& \left\|\left[\mathrm{b}, \mathcal{J}_{\beta}^{\mathrm{L}}\right](\mathrm{f})\right\|_{\mathrm{LM}_{\mathrm{q}^{\prime}, \varphi_{2}}^{\alpha, v}} \lesssim \sup _{\mathrm{r}>0}\left(1+\frac{\mathrm{r}}{\rho(e)}\right)^{\alpha} \varphi_{2}(\mathrm{r})^{-1} \mathrm{r}^{-\mathrm{Q} / \mathrm{q}}\left\|\left[\mathrm{b}, \mathcal{J}_{\beta}^{\mathrm{L}}\right](\mathrm{f})\right\|_{\mathrm{L}_{\mathrm{q}}(\mathrm{B}(e, \mathrm{r}))} \\
& \lesssim[b]_{\theta} \sup _{\mathrm{r}>0}\left(1+\frac{\mathrm{r}}{\rho(e)}\right)^{\alpha} \varphi_{2}(\mathrm{r})^{-1} \int_{2 \mathrm{r}}^{\infty}\left(1+\ln \frac{\tau}{\mathrm{r}}\right) \frac{\|\mathrm{f}\|_{\mathrm{L}_{\mathrm{p}}(\mathrm{B}(e, \tau))}}{\tau^{\frac{Q}{q}}} \frac{\mathrm{d} \tau}{\tau} \lesssim[\mathrm{b}]_{\theta}\|f\|_{\mathrm{LM}_{\mathcal{P}, \varphi_{1}}^{\alpha, V}} .
\end{aligned}
$$

Remark 3.7. Note that, Theorem 3.2 in the case of $V \equiv 0$ was proved in [18, Theorem 5.2]. 


\section{References}

[1] A. Akbulut, R. V. Guliyev, S. Celik, M. N. Omarova, Fractional integral associated with Schrödinger operator on vanishing generalized Morrey spaces, accepted in J. Math. Ineq., (2018). 1, iv

[2] A. Akbulut, V. S. Guliyev, M. N. Omarova, Marcinkiewicz integrals associated with Schrödinger operators and their commutators on vanishing generalized Morrey spaces, Bound. Value Probl., 2017 (2017) 16 pages. 1, iv, 2.2, $2.8,2.9$

[3] J. Alvarez, J. Lakey, M. Guzman-Partida, Spaces of bounded $\lambda$-central mean oscillation, Morrey spaces, and $\lambda$-central Carleson measures, Collect. Math., 51 (2000), 1-47. i

[4] B. Bongioanni, E. Harboure, O. Salinas, Commutators of Riesz transforms related to Schödinger operators, J. Fourier Anal. Appl., 17 (2011), 115-134. 1, 2.3, 2.4

[5] T. Bui, Weighted estimates for commutators of some singular integrals related to Schrödinger operators, Bull. Sci. Math., 138 (2014), 270-292. 2.5

[6] L. Capogna, D. Danielli, S. D. Pauls, J. T. Tyson, An introduction to the Heisenberg group and the sub-Riemannian isoperimetric problem, Birkhuser Verlag, Basel, (2007). 1

[7] G. Di Fazio, M. A. Ragusa, Interior estimates in Morrey spaces for strong solutions to nondivergence form equations with discontinuous coefficients, J. Funct. Anal., 112 (1993), 241-256. 1

[8] A. Eroglu, J. V. Azizov, A note of the fractional integral operators in generalized Morrey spaces on Heisenberg groups, Trans. Natl. Acad. Sci. Azerb. Ser. Phys.-Tech. Math. Sci., 37 (2017), 86-91. 1, 2.8, 2.9

[9] A. Eroglu, V. S. Guliyev, C. V. Azizov, Characterizations for the fractional integral operators in generalized Morrey spaces on Carnot groups, Math. Notes, 102 (2017), 127-139. 1

[10] D. S. Fan, S. Z. Lu, D. C. Yang, Boundedness of operators in Morrey spaces on homogeneous spaces and its applications, Acta Math. Sinica (Chin. Ser.), 14 (1998), 625-634. 1

[11] G. B. Folland, E. M. Stein, Estimates for the $\partial_{\mathrm{b}}$-complex and analysis on the Heisenberg group, Comm. Pure Appl. Math., 27 (1974), 429-522. 1

[12] G. B. Folland, E. M. Stein, Hardy Spaces on Homogeneous Groups, Princeton University Press, Princeton, (1982). 1, 2

[13] V. S. Guliyev, Boundedness of the maximal, potential and singular operators in the generalized Morrey spaces, J. Inequal. Appl., 2009 (2009), 20 pages. 3

[14] V.S. Guliyev, Function spaces and integral operators associated with Schrödinger operators: an overview, Proc. Inst. Math. Mech. Natl. Acad. Sci. Azerb., 40 (2014), 178-202. 1, iv

[15] V. S. Guliyev, Function spaces, integral operators and two weighted inequalities on homogeneous groups. Some applications, Elm, Baku, (1999). iii, 3

[16] V. S. Guliyev, Generalized local Morrey spaces and fractional integral operators with rough kernel, J. Math. Sci. (N.Y.), 193 (2013), 211-227. iii, 3

[17] V. S. Guliyev, Integral operators on function spaces on the homogeneous groups and on domains in $\mathbb{R}^{n}$, Doctors degree dissertation, Mat. Inst. Steklov, Moscow, (1994). iii, 3

[18] V. S. Guliyev, A. Eroglu, Y. Y. Mammadov, Riesz potential in generalized Morrey spaces on the Heisenberg group, J. Math. Sci. (N. Y.), 189 (2013), 365-382. iii, 1, 3.7

[19] V. S. Guliyev, T. S. Gadjiev, S. Galandarova, Dirichlet boundary value problems for uniformly elliptic equations in modified local generalized Sobolev-Morrey spaces, Electron. J. Qual. Theory Differ. Equ., 2017 (2017), 17 pages. iii

[20] V. S. Guliyev, R. V. Guliyev, M. N. Omarova, M. A. Ragusa, Schrödinger type operators on local generalized Morrey spaces related to certain nonnegative potentials, (submitted). 1, iv, 2.7, 2.9

[21] V. S. Guliyev, Y. Y. Mammadov, Boundedness of the fractional maximal operator in generalized Morrey space on the Heisenberg group, Indian J. Pure Appl. Math., 44 (2013), 185-202. 1

[22] V. S. Guliyev, M. N. Omarova, M. A. Ragusa, A. Scapellato, Commutators and generalized local Morrey spaces, J. Math. Anal. Appl., 457 (2018), 1388-1402. iii

[23] H. Q. Li, Estimations $\mathrm{L}_{p}$ des oprateurs de Schrödinger sur les groupes nilpotents, J. Funct. Anal., 161 (1999), $152-218$. $2.1,3,3,3$

[24] C. B. Morrey, On the solutions of quasi-linear elliptic partial differential equations, Trans. Amer. Math. Soc., 43 (1938), 126-166. i, 1

[25] S. Polidoro, M. A. Ragusa, Sobolev-Morrey spaces related to an ultraparabolic equation, Manuscripta Math., 96 (1998), 371-392. 1

[26] Z. Shen, $\mathrm{L}_{p}$ estimates for Schrödinger operators with certain potentials, Ann. Inst. Fourier (Grenoble), 45 (1995), $513-$ 546. 1

[27] E. M. Stein, Harmonic Analysis: Real-variable methods, orthogonality, and oscillatory integrals, Princeton University Press, Princeton, (1993). 2, 3, 3, 3

[28] L. Tang, J. Dong, Boundedness for some Schrödinger type operator on Morrey spaces related to certain nonnegative potentials, J. Math. Anal. Appl., 355 (2009), 101-109. 1, ii

[29] R. Wheeden, A. Zygmund, Measure and integral: An introduction to real analysis, Marcel Dekker, New York-Basel, (1977). 2.6 